\title{
Análise comparativa entre dois programas de prevenção e reabilitação cardiovascular
}

\author{
José Henrique Ramos e Osni Jacó da Silva \\ Universidade Federal de Santa Catarina
}

\section{RESUMO}

Este estudo foi realizado para investigar os procedimentos metodológicos empregados por dois Programas de Prevenção e Reabilitação Cardiovascular (PPRC), que atuam na fase III e IV, em Florianópolis. O objetivo básico do estudo foi investigar as diferenças de metodologia proposta pelos programas. Procurou-se identificar se eles possibilitam a autosuficiência/independência de seus pacientes. Houve observação sistemática e entrevista com os responsáveis pelos programas. As conclusões foram de que existem pequenas diferenças na metodologia empregada entre os dois programas, tais como a duração e as fases da sessão e a intensidade do estímulo. Apenas um dos programas estimula a auto-suficiência/independência dos pacientes quanto à continuidade dos exercícios físicos.

Palavras-chave: Programas de reabilitação cardíaca. Treinamento de pacientes cardiopatas. Exercício físico. Exercício e saúde. Doença cardiovascular. Doença arterial coronariana.

\section{ABSTRACT \\ Comparative analisys between two programs of cardiovas- cular prevention and rehabilitation}

This study aims at investigating methodological procedures used by two Programs of Cardiovascular Prevention and Rehabilitation (PCPR) performed in the III and IV phases in Florianópolis. The main objective of this study was to investigate the differences of methodology proposed by the programs. The authors tried to identify if they allow the selfsufficiency/independency of their patients. There was a sys-

Endereço para correspondência:

Prof. Osni Jacó da Silva

Universidade Federal de Santa Catarina

Centro de Desportos

Departamento de Educação Física

Campus Universitário - Trindade

88040-900 - Florianópolis, SC - Brasil

Fones: (048) 331-9834 e 9721770 cel - Fax: (048) 3319927

e-mail: osni@cds.ufsc.br tematic observation and also an interview with the people who are responsible for the programs. The conclusions show that there are small differences in the methodology used in the two programs, such as the length and the phases of the session and the intensity of the stimulus. Only one of the programs stimulates the patients' self-sufficiency/independency concerning the maintenance of the physical exercises.

Key words: Cardiac rehabilitation programs. Exercise training of cardiac patients. Physical exercise. Exercise and health. Cardiovascular disease. Coronary heart disease.

\section{INTRODUÇÃO}

A reabilitação cardiovascular ( $\mathrm{RC})$, hoje em dia, vem sendo muito empregada e defendida por vários autores. Exemplo disso é a referência feita por Meneghelo et al. ${ }^{1}$ ao citarem que "a mobilização precoce e controlada dos doentes infartados, 24h após o desaparecimento dos sintomas ou controle de complicações, minimiza a ocorrência dos malefícios do repouso prolongado".

Sabe-se atualmente que o repouso prolongado, após acometimento cardiovascular, é prejudicial ao funcionamento fisiológico do organismo, prova disso é o que Pollock et al. ${ }^{2}$ afirmam: "Um rígido repouso no leito tem acentuadas influências deletérias sobre o funcionamento fisiológico e bastam alguns dias ou semanas para que o paciente tenha sua capacidade cardiorrespiratória, volume sanguíneo, contagem de hemácias, nitrogênio e balanço protéico, força e flexibilidade significativamente diminuídas, bem como aumentados os riscos de trombo-embolismo e hipotensão ortostática."

Portanto, o paciente deve ser submetido à reabilitação tão logo seja possível, após ter tido complicações cardiovasculares e não ter ido a óbito.

O programa de reabilitação cardíaca, segundo Pollock et al. “ “...pode ser considerado como o processo de restauração das funções psicológicas, físicas e sociais em indivíduos com manifestação prévia de doença arterial coronária (DAC), a níveis ótimos." Alfieri \& Duarte ${ }^{3}$ completam afirmando que o treinamento físico é parte integrante da RC. Atualmente, o conceito de reabilitação inclui prevenção, diagnóstico, cui- 
dados médicos e cirúrgicos, provisão de serviços de saúde, educação, treinamento e outros meios para aumentar a capacidade funcional.

No que diz respeito à finalidade da $\mathrm{RC}$, há consenso de que tenha como objetivo final o restabelecimento da condição física, psíquica e social, em níveis anteriores às complicações para que o paciente retorne a sua ótima condição clínica, fisiológica, psicológica, social e profissional. Enfim, visa melhorar e ampliar a qualidade de vida do paciente cardiopata, permitindo um nível mais elevado de atividade física, compatível com a extensão da doença.

Muitos podem ser os benefícios de um Programa de Prevenção e Reabilitação Cardiovascular (PPRC). Segundo Leite $^{4}$, o sistema cardiovascular sofre modificações significativas após treinamento físico e essas alterações ocorrem fisiológica e anatomicamente, "afetando o sistema de transporte, extração e utilização de oxigênio”. Por exemplo, segundo Pollock et al. ${ }^{2}$, em pacientes submetidos à cirurgia de revascularização do miocárdio, "a atividade física pode não só ajudar a diminuir a rigidez pós-cirúrgica mas também prevenir complicações como, por exemplo, a atelectasia pós-operatória". E acrescenta que outros benefícios incluem "a diminuição da incidência e intensidade da depressão e ansiedade, bem como alta hospitalar mais precoce".

Ainda, sobre o mesmo assunto, Pozzan et al. ${ }^{5}$ afirmam que "a atividade física regular é o princípio básico de um programa de reabilitação cardíaca” e que, se o exercício físico foi realizado com metodologia segura, é capaz de produzir alterações significativas. Os autores concluem afirmando que, dessa forma, o exercício físico contribui para a terapêutica empregada no controle, prevenção e reabilitação dos coronarianos.

Em decorrência dos grandes benefícios obtidos pela prevenção e reabilitação, existem em países desenvolvidos, como a Alemanha e os Estados Unidos, e inclusive em países "menos desenvolvidos", como Cuba, investimentos e conseqüentes progressos, muito importantes. Só para exemplificar, segundo Silva \& Silva ${ }^{6}$, a Alemanha destina cerca de $3 \%$ de toda a verba para a Previdência Social para esse tipo de programa de reabilitação.

\section{OBJETIVOS}

Identificar se existiam diferenças nos procedimentos metodológicos e quanto à capacitação dos pacientes para a autosuficiência/independência para a prática de exercícios físicos, em dois Programas de Prevenção e Reabilitação Cardiovascular.

\section{METODOLOGIA}

A população desta pesquisa foi composta pelos Programas de Prevenção e Reabilitação Cardíaca: 1) da Academia
Catarinense de Ginástica (CRPC) e, 2) do Núcleo de Cardiologia e Medicina Desportiva do Centro de Educação Física e Desportos (Cefid) da Udesc.

Os entrevistados, intencionalmente selecionados, foram os responsáveis por ambos os programas.

Também foi realizada observação sistemática das sessões de uma semana de atividade.

\section{RESULTADOS E DISCUSSÃO}

\section{A metodologia dos programas}

Os programas apresentavam metodologia semelhante e uma estrutura de sessão, conforme preconizada por Alfieri ${ }^{7}$ e Meneghelo et al. ${ }^{1}$, que consiste em aquecimento, estímulo ou treinamento efetivo e desaquecimento. Porém, em relação ao tempo destinado a cada uma dessas etapas, havia pequenas diferenças, pouco significativas. Em ambos, o tempo do aquecimento era em torno de 10 minutos, ocupado com alongamento muscular e aquecimento articular.

Em relação ao estímulo cardiovascular, o programa do Cefid destinava 40 minutos e o CRPC, entre 30 e 40 minutos. Para isso, ambos utilizavam a caminhada e a corrida. Esta última era praticada apenas quando era possível aos pacientes.

Quanto ao "resfriamento" ou "volta à calma", o Cefid destinava em torno de 10 minutos, enquanto o CRPC, 5 minutos. Ambos utilizavam alongamentos e relaxamento nesta etapa. Alfieri ${ }^{7}$ preconiza de 5 a 10 minutos para essa etapa.

Nessa questão o que há de diferente mesmo entre os programas, e que eleva o tempo total da sessão no CRPC para 1:15h, contra a 1:00h do Cefid, é a inclusão de uma etapa (de aproximadamente 15 minutos) logo após o fim da fase de estímulo, que consiste de ginástica localizada (segundas e quartas-feiras), inclusive com utilização de steps, para o desenvolvimento e manutenção de força e, nas sextas-feiras, consiste de sessões de ginástica para reeducação postural.

No programa do Cefid, qualidades como resistência muscular, flexibilidade, relaxamento, equilíbrio e coordenação são contempladas durante o aquecimento, ao contrário do CRPC, que o fazia durante o "resfriamento". O programa do Cefid a cada duas semanas, em forma de rodízio, prioriza uma qualidade física: resistência muscular, flexibilidade, relaxamento, equilíbrio e coordenação. Todas essas atividades que visam aumentar força, flexibilidade e equilíbrio são importantes à medida que proporcionam melhoras na capacidade funcional, permitindo "mobilidade, independência, capacidade para desempenhar os auto-cuidados necessários, referentes ao repouso, sono, capacidade para participar em atividades ocupacionais e de recreação"».

Quanto ao tipo de exercício, ambos utilizam a caminhada e a corrida (quando necessária e possível) em sua fase de estímulo. Isso porque há pacientes que só atingem a freqüência cardíaca de treinamento (FCT), ou como Godoy ${ }^{9}$ con- 
venciona, frequiência cardíaca de equilíbrio, correndo, pois a caminhada não estimula suficientemente o sistema cardiorrespiratório, ficando, então, a freqüência cardíaca abaixo da faixa preconizada. Nos dois programas, isso acontece com um número reduzidíssimo de pacientes, normalmente mais jovens ou com melhor condicionamento físico. Sobre o tipo de exercício, há na literatura consenso entre os autores de que o exercício aeróbio é o mais recomendado.

Quanto à freqüência semanal, ambos utilizavam três sessões por semana, o que está de acordo com a literatura pesquisada.

Meneghelo et al. ${ }^{1}$ afirmam que a freqüência deve ser, no mínimo, de duas vezes por semana, mas que a maioria dos programas inclui três sessões semanais. A mesma freqüência semanal, de três sessões, também é defendida por Alfieri ${ }^{3}$ e Benchimol et al. ${ }^{10}$.

Em relação à intensidade da carga de trabalho, a literatura afirma que deve ser prescrita através de um teste de esforço (TE) ergométrico individual prévio ${ }^{9}$. Comprovou-se que os dois programas preconizam a realização do teste para seus pacientes. Ambos solicitam que os testes sejam máximos, dada a sua finalidade.

Porém, no CRPC, constatou-se que alguns pacientes com baixos fatores de risco ingressaram no programa e, até aquele momento, não haviam realizado o teste; este era o caso de uma pequena parcela dos pacientes.

No Cefid, segundo o entrevistado, todos os pacientes em exercício realizaram o TE máximo para a prescrição da intensidade dos exercícios. Segundo Godoy ${ }^{9}$, o TE está sempre indicado para indivíduos aparentemente saudáveis, com fator de risco cardiovascular e acima dos 45 anos de idade. Para indivíduos entre 35 e 44 anos, a indicação é individual e não obrigatória. Ainda segundo Godoy ${ }^{11}$, "o teste de esforço é um método importante na programação do condicionamento físico e fundamental para a reabilitação cardíaca."

Feito o teste de esforço, é necessário prescrever a intensidade do estímulo. Sobre a intensidade, Godoy ${ }^{9}$ afirma que é a variável fundamental num programa.

O programa CRPC prescreve o exercício numa faixa entre 60 e $70 \%$ da frequiência cardíaca máxima (FCM), exceto para um paciente de 34 anos, que se exercita em intensidade entre 70 e $80 \%$ da FCM.

O programa do Cefid trabalha com uma faixa mais ampla, entre 60 e $80 \%$ da FCM, para todos os seus pacientes. No início o paciente faz seus exercícios com intensidade próxima ao limite inferior da faixa $(60 \%)$ e, no decorrer do programa, vai tendo essa intensidade aumentada, até chegar no limite superior da faixa $(80 \%)$. Nesse programa, na primeira semana de exercício (chamada consulta de campo), não há preocupação, ainda, com a intensidade; afinal, o paciente está em fase de adaptação ao programa.

Em relação à intensidade do exercício, Hellerstein \& Franklin ${ }^{\text {apud } 3}$, consideram como ótima para o exercício, 57 a $78 \%$ do $\mathrm{VO}_{2}$ máx., que corresponde a 70 e $85 \%$ da FCM. Eles afirmam que "nestes limites há uma série de evidências de que o metabolismo aeróbio está sendo estimulado...". Ainda segundo os mesmos autores, a intensidade não deve ser exaustiva para obter uma resposta substancial.

Para Alfieri et al. ${ }^{3}$, a intensidade para indivíduos com baixo condicionamento (sedentários) deve-se situar em torno de $60 \%$ da FCM prevista (que corresponde a $45 \%$ do $\mathrm{VO}_{2}$ máx.). Os autores afirmam que é desconhecido o limite máximo para o treinamento, porém McArdle et al. ${ }^{\text {apud } 3}$ preconizam que o limite superior fique em torno de $90 \%$ da FCM (ou $85 \%$ do $\mathrm{VO}_{2}$ máx.).

No entanto, Pozzan et al. ${ }^{5}$ fazem menção a um programa de reabilitação em que a intensidade prescrita nunca ultrapassou $85 \%$ da FCM.

Leite $^{4}$ preconiza a intensidade entre 60 e $85 \%$ da FCM (ou 50 a $70 \%$ do $\mathrm{VO}_{2}$ máx.) para que o treinamento surta os efeitos desejados. Afirma também, que se a intensidade for inferior a $60 \%$ da FCM, pouco efeito de treinamento será obtido.

Para a contagem da FC durante os exercícios, os dois programas utilizam meios diferentes. Mellerowicz \& Meller ${ }^{12}$ descrevem as duas formas utilizadas pelos programas: a contagem da FC durante ou imediatamente após o término do exercício, pelo próprio paciente ou através da utilização de aparelhos específicos que emitem um sinal quando a FC excede o limite máximo.

No programa CRPC (programa de uma instituição privada) são utilizados monitores de freqüência cardíaca por todos os pacientes. Dessa forma, o professor de Educação Física somente questiona ao paciente, e este observa sua FC no equipamento instalado em seu pulso (unidade receptora) e informa ao professor que faz o controle da intensidade.

No programa do Cefid, a contagem da FC é feita manualmente, através da palpação do pulso radial, normalmente realizada pelos próprios pacientes. Em momentos predeterminados, são emitidos sinais sonoros (por apito) por um monitor. Nesses momentos, os pacientes interrompem momentaneamente o exercício (caminhada ou corrida) e conferem as suas frequiências cardíacas. Logo em seguida, informam ao monitor responsável sua $\mathrm{FC}$ e este, então, faz o controle da intensidade do exercício.

Em relação à duração total do programa, verificou-se que não há um tempo determinado. Isso ocorre até mesmo porque ambos os programas fazem a prevenção e a reabilitação cardíaca, ou seja, atuam em fase III e IV da reabilitação cardiovascular.

Sobre a terceira fase da reabilitação, Alfieri et al. $^{3}$ afirmam que essa etapa "corresponde aos programas a longo prazo". Isso porque esses autores juntam a fase IV à fase III, diferentemente de outros autores, como Meneghelo et al. ${ }^{1}$. Estes últimos preconizam algo em torno de 12 meses para a fase III e prazo indeterminado para a fase IV (ou de manutenção). 


\section{Os objetivos dos programas}

Grosso modo, os objetivos dos dois programas são parecidos. Entretanto, julgamos conveniente ressaltar algumas diferenças.

Com relação ao CRPC, em que este programa difere de outros é o pequeno número de pacientes por turma (máximo 15) e a fase da sessão, que objetiva desenvolvimento e manutenção de força útil (segundas e quartas-feiras) e reeducação postural (sextas-feiras) com duração aproximada de 15 minutos, destinada exclusivamente aos objetivos citados logo acima.

Quanto ao número de pacientes nas sessões, Alfieri et al. ${ }^{3}$ são alguns dos poucos autores a se manifestar sobre essa questão. Eles fazem menção a um programa convencional de reabilitação, existente no Instituto Dante Pazzanese de Cardiologia, em São Paulo, onde até 200 pacientes se submetem à reabilitação em grupos de 13 .

No que diz respeito ao módulo da sessão, dedicado exclusivamente à força útil e reeducação postural, Leite ${ }^{4}$ afirma que os programas devem conter treinamento para o desenvolvimento e manutenção, entre outros, de flexibilidade e força muscular. Afirmam que isso serve para prevenir lesões músculo-esqueléticas, corrigir postura e proporcionar melhoras na coordenação.

Arakaki \& Magalhães ${ }^{13}$ citam uma das fases da sessão, composta de exercícios calistênicos para estimular a coordenação e melhorar a flexibilidade articular, através de atividades de solo e ortostáticas.

\section{CONCLUSÕES}

O objetivo propriamente dito dos programas é o mesmo: reabilitar o paciente e prevenir as enfermidades cardíacas, além de outras doenças degenerativas.

No entanto, a diferença é o modo como isso se dá. Devese fazer a reabilitação capacitando os pacientes para a independência? Ou deve-se somente oferecer um espaço com estrutura e orientação especializada e cobrar por isso?

Somente um dos programas, o do Cefid, possibilita a autosuficiência/independência de seus pacientes.

Essa é, sem dúvida, a diferença mais significativa entre os programas observados. Um deles, o Cefid, é mantido por uma instituição de ensino pública, a Universidade do Estado de Santa Catarina - Udesc. O outro, CRPC, da Academia Cata- rinense de Ginástica, é mantido por uma instituição de ensino privada, de primeiro e segundo graus, o Colégio Catarinense. Dessa forma, são programas oferecidos por instituições de caráter diferente e, conseqüentemente, com alguns objetivos diferentes.

$\mathrm{O}$ que é privado costuma ter como filosofia atender aos interesses particulares, dos proprietários. E o que é público, como o próprio termo expressa, tende a atender aos interesses da sociedade como um todo e não a apenas uma fração privilegiada dela. Se analisarmos bem a questão, concluiremos que, se os serviços essenciais, como os de saúde e educação fossem todos de caráter público, não haveria necessidade dos privados. Isso é o que ocorre em países do Primeiro Mundo, como, por exemplo, o Canadá.

\section{REFERÊNCIAS}

1. Meneghelo RS, Ferraz AS, Ghorayeb N. Reabilitação e atividade esportiva após infarto do miocárdio. Rev Soc Cardiol Est São Paulo 1993;3: 86-91.

2. Pollock ML, Wilmore JH, Fox SM. Exercícios na saúde e na doença. Rio de Janeiro: Medsi, 1993.

3. Alfieri RG, Duarte GM. Marcondes, exercício e o coração. Rio de Janeiro: Cultura Médica, 1993.

4. Leite PF Sistema cardiovascular, treinamento físico e reabilitação cardíaca. J Bras Med 1984;46:98-117.

5. Pozzan R, Cruz PDM, Castier MB, Barbosa EC, Barbosa JSO, Rocha PJ, et al. Reabilitação cardíaca em coronariopatas. Avaliação após 3 e 6 meses de treinamento aeróbio em nível comunitário. Arq Bras Cardiol 1988;50:305-10.

6. Silva OJ, Silva, TJC. Exercício e saúde: fatos e mitos. Florianópolis: EdUFSC, 1995.

7. Alfieri RG. Exercício físico. Arq Bras Cardiol 1995;55:221-2.

8. Trentini M, Silva DGV, Martins CR, Antônio MC, Tomaz CE, Duarte R. Qualidade de vida dos indivíduos com doenças cardiovasculares crônicas e diabetes mellitus. Rev Gaucha Enferm 1995;11:20-7.

9. Godoy M. Prescrição de exercícios. Fitcor em revista, edição especial, 1997:19-23.

10. Benchimol CB, Barbosa Filho J, Benchimol AB. O exercício e o coração. J Bras Med 1997;45:103-7.

11. Godoy M. Reabilitacão cardíaca. Fitcor em revista, edição especial, 1997:44-52.

12. Mellerowicz H, Meller W. Bases fisiológicas do treinamento físico. São Paulo: Editora da USP, 1997.

13. Arakaki H, Magalhães HM. Programas supervisionados em reabilitação cardiovascular - abordagem de prescrição de exercício. Rev Soc Cardiol Est São Paulo 1996;6:23-30. 